\title{
NANOESTRUTURAS DE CARBONO (NANOTUBOS, GRAFENO): QUO VADIS?
}

\author{
Aldo J. G. Zarbin* \\ Departamento de Química, Universidade Federal do Paraná, CP 19081, 81531-990 Curitiba - PR, Brasil \\ Marcela M. Oliveira \\ Departamento Acadêmico de Química e Biologia, Universidade Tecnológica Federal do Paraná, 81280-340 Curitiba - PR, Brasil
}

Recebido em 16/7/13; aceito em 1/10/13; publicado na web em 24/10/13

\begin{abstract}
CARBON NANOSTRUCTURES (NANOTUBES AND GRAPHENE): QUO VADIS? We describe general considerations about the present and the future standing of carbon nanostructures, mainly carbon nanotubes and graphene. Basic concepts and definitions, select structure/property relationships, and potential applications are reviewed. The analysis of the global market for these nanostructures, the commercial products available currently, the role of the chemistry, the main challenges remaining and a brief view of the field in Brazil are also presented and discussed.
\end{abstract}

Keywords: carbon nanotubes; graphene; nanoscience and nanotechnology.

\section{INTRODUÇÃO}

A última década do século XX foi marcada pela consolidação e crescimento vertiginoso de uma nova área do conhecimento, que trouxe no seu bojo a promessa de uma verdadeira revolução científica e tecnológica, derrubando antigos paradigmas, abrindo novos horizontes e rompendo fronteiras estabelecidas entre disciplinas: a nanociência e nanotecnologia $(\mathrm{N} \& \mathrm{~N})$. De maneira bastante simplificada, o princípio básico que suporta a $\mathrm{N} \& \mathrm{~N}$ se baseia no fato de que propriedades da matéria dependem não só da sua composição e estrutura, como também de seu tamanho e formato. Abaixo de um determinado tamanho crítico, sempre na ordem de alguns nanômetros (daí o termo nanociência e nanotecnologia - a ciência e tecnologia da matéria em escala nanométrica), as propriedades de um determinado material se tornam diferenciadas daquelas observadas para o mesmo material em escala macroscópica. Desta forma, novos materiais, com propriedades diferenciadas, passaram a ser preparados somente pelo controle de tamanho e forma de materiais já conhecidos, ampliando significativamente o leque de oportunidades. ${ }^{1} \mathrm{~A} N \& \mathrm{~N}$ se caracteriza por uma alta multi- e interdisciplinaridade, na qual diferentes especialidades naturalmente passaram a sinergicamente compartilhar suas expertises: químicos, físicos, engenheiros, biólogos, matemáticos, médicos, ambientalistas, advogados, dentre outros, possibilitaram a compreensão de fenômenos e a geração de produtos com aplicações em potencial em todos os ramos da atividade humana: energia, agricultura, meio ambiente, bens de consumo, saúde e medicina, manufatura, construção civil, transporte, eletrônica, tecnologia da informação, cosméticos, têxteis, entretenimento, comunicação, lazer, etc.

Dentre todos os novos(?) materiais oriundos da $\mathrm{N} \& \mathrm{~N}$, talvez os mais sintomáticos são os conhecidos genericamente como nanomateriais de carbono, dos quais os nanotubos de carbono (NTC), e mais recentemente o grafeno, são os mais representativos. Não deixa de ser fascinante a constatação de que o elemento químico símbolo da vida e massivamente associado à ciência química seja o único constituinte de alguns dos materiais mais intrigantes conhecidos. A riqueza e diversidade do elemento carbono, tão familiar aos químicos orgânicos, se manifesta também na química de materiais e na N\&N.

A Figura 1 apresenta algumas estruturas alótropas do carbono atualmente conhecidas. Há não muito tempo atrás, esta figura estaria

*e-mail: aldozarbin@ufpr.br limitada às estruturas do grafite e do diamante, muito embora diversas formas de carbono amorfo (negro de fumo, carvão, carbono vítreo, etc.) fossem conhecidas, mas estruturalmente pouco compreendidas. O grafite é um exemplo clássico de sólido com estrutura lamelar, onde cada átomo de carbono com hibridização $\mathrm{sp}^{2}$ está ligado a outros três átomos, formando folhas bidimensionais com a aparência de uma colmeia. ${ }^{2}$ Cada uma destas folhas individuais recebe o nome de folha de grafeno, e o empilhamento das mesmas, via atrações de van der Waals (através dos elétrons nos orbitais p puros de cada átomo de carbono), confere a estrutura tridimensional do grafite (Figura 1a). O diamante, por sua vez, é um sólido covalente formado por átomos de carbono com hibridização $\mathrm{sp}^{3}$, ligados a quatro outros átomos de carbono, em uma geometria tetraédrica $^{2}$ (Figura 1b). Em 1985 foi descrita uma nova família de alótropos de carbono, os fulerenos, ${ }^{3}$ que ao contrário dos outros alótropos, correspondem a uma forma molecular de carbono. Os fulerenos são moléculas nanométricas esferoidais constituídas somente por átomos de carbono com hibridização $\mathrm{sp}^{2}$ e cujo representante mais famoso é o buckminsterfulereno, $\mathrm{C}_{60}$ (Figura 1c). ${ }^{4} \mathrm{O}$ prêmio Nobel de química de 1996 foi agraciado aos Professores Robert F. Curl Jr, Harold W. Kroto e Richard E. Smalley, pela descoberta dos fulerenos. (a)

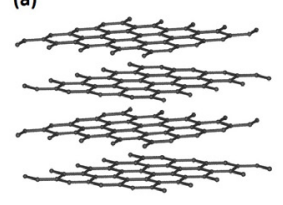

(d)

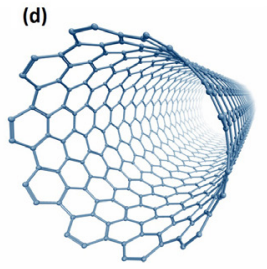

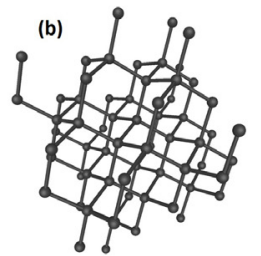

(e)

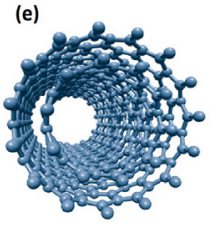

(c)
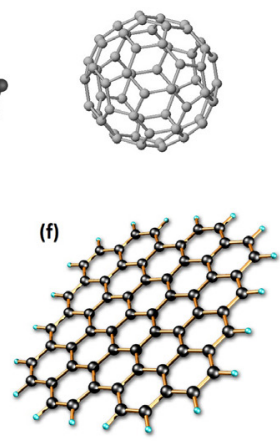

Figura 1. Representação esquemática de diferentes alótropos de carbono: a) grafite; b) diamante; c) fulereno; d) nanotubo de carbono de parede simples; e) nanotubo de carbono de parede múltipla; f) grafeno

A estrutura dos nanotubos de carbono pode ser visualizada e idealizada a partir do enrolamento de uma folha de grafeno, como 
esquematicamente representado na Figura 1d. Embora houvesse relatos de sua preparação em um artigo russo de $1958^{5}$ e em outro artigo de um grupo japonês de $1976,{ }^{6}$ a comunidade considera o trabalho de Iijima, em $1991,{ }^{7}$ como sendo a primeira descrição deste material (embora o termo nanotubo de carbono só venha a aparecer um ano depois, em artigo do mesmo Iijima em parceria com Ajayan). ${ }^{8}$ Esta descrição foi extremamente importante, pois além da descrição do material, há um estudo estrutural completo, o que não havia sido realizado nas descrições anteriores.

O termo "nanotubo de carbono" corresponde, na verdade, a uma grande família de materiais com características diferentes entre si, formados a partir do enrolamento de uma ou mais folhas de grafeno a partir do seu próprio eixo, formando estruturas cilíndricas (tubulares) com diâmetros na faixa dos nanômetros (nanotubos), e comprimentos que variam de alguns micrometros até vários centímetros. Em um primeiro momento, os nanotubos podem ser separados em dois grupos: os nanotubos de carbono de paredes simples (Figura 1d), no qual uma única folha de grafeno é responsável pela sua estrutura; e os nanotubos de carbono de paredes múltiplas (Figura 1e), onde várias folhas de grafeno se enrolam de forma concêntrica, como um tubo coaxial, separadas entre si por uma distância muito parecida com aquela observada entre as folhas de grafeno no grafite. ${ }^{9}$

A beleza da estrutura de um nanotubo de carbono é diretamente responsável por suas propriedades, algumas delas bastante imperativas: ${ }^{9}$

i) a ligação $\mathrm{C}_{\mathrm{sp} 2} \mathrm{C}_{\mathrm{sp} 2}$ é a mais forte existente na natureza, o que confere uma resistência extremamente elevada para os nanotubos de carbono, com módulos de Young medidos em nanotubos individuais na faixa de TPa, mais de 10 vezes superiores às fibras de carbono, por exemplo. Esta resistência é associada a uma rara propriedade de flexibilidade, pois os nanotubos podem ser dobrados, tensionados e flexionados sem que haja destruição de sua estrutura;

ii) nanotubos de carbono de paredes simples podem apresentar características metálicas ou semicondutoras, ${ }^{10}$ com diferentes valores de band gap, de acordo com o diâmetro e a orientação pela qual a folha de grafeno foi enrolada em relação ao eixo do tubo (estas diferentes orientações são conhecidas como "quiralidades", mas não têm nenhuma relação com o termo comumente utilizado pelos químicos para designar moléculas com diferentes orientações espaciais). Trata-se da primeira observação de modulação de propriedades elétricas e de estruturas de banda de um material por fatores eminentemente geométricos. Nanotubos de paredes múltiplas por sua vez sempre apresentam condutividade metálica;

iii) a estrutura curva com diâmetro reduzido dos nanotubos confina os elétrons da folha de grafeno em uma única dimensão (o comprimento do tubo). Este confinamento unidimensional modifica a estrutura de bandas dos nanotubos, criando níveis discretos conhecidos como "singularidades de van Hove", onde a possibilidade de transições entre estas singularidades lhes confere propriedades óticas únicas, que também são dependentes da 'quiralidade' e do diâmetro dos nanotubos (uma vez que estes fatores alteram a estrutura de bandas - e as energias das singularidades de van Hove - dos nanotubos individuais) ${ }^{10}$

iv) a condutividade térmica dos nanotubos de carbono é uma das mais altas conhecidas, excedendo bastante a observada para o diamante, por exemplo;

v) a estrutura perfeita de um nanotubo apresenta átomos de carbono com ligações incompletas nas extremidades abertas (dangling bonds), sendo portanto pontos de alta reatividade. Entretanto, amostras reais possuem defeitos nas paredes laterais dos nanotubos (ligações incompletas, presença de heteroátomos, presença de pentágonos e heptágonos na estrutura, átomos de carbono com hibridização $\mathrm{sp}^{3}$ ligados a grupos funcionais, etc.), o que afeta suas propriedades, e possibilita a realização de uma série de modificações químicas a partir de reações nestes defeitos estruturais. Desta forma, novas funcionalidades podem ser adicionadas ao material; ${ }^{11,12}$

vi) nanotubos possuem uma cavidade oca, que pode ser utilizada como template para o crescimento de diferentes tipos de materiais (metais, óxidos, fulerenos, polímeros, etc.), propiciando a possibilidade de formação de nanocompósitos com propriedades sinergísticas, além da introdução de novas propriedades aos nanotubos (por exemplo, propriedades magnéticas, pelo crescimento de um nanofio magnético no interior das cavidades dos nanotubos)..$^{13,14}$

Todas estas características em conjunto, em uma única classe de material, levaram a uma miríade de possibilidades de aplicação em potencial de nanotubos de carbono, em vários sistemas, produtos e dispositivos, fazendo com que seja considerado um material-chave da N\&N. Estes dados, somado a uma relação estrutura/propriedade inédita até então, fez com que este nanomaterial passasse a ser estudado por cientistas de várias áreas (químicos, físicos, engenheiros, matemáticos, biólogos, médicos, agrônomos, cientistas ambientais, etc.), interessados em aprofundar diferentes aspectos do conhecimento básico, bem como em possibilidades reais de aplicações; fez também crescer de forma importante o interesse de indústrias e do setor produtivo em geral, à procura de novos produtos e de substituição de componentes em produtos já existentes. O número de artigos científicos publicados sobre diferentes aspectos de síntese, caracterização, propriedades e aplicações de materiais envolvendo nanotubos de carbono impressiona: em 08/07/2013 uma busca no Web of Science com a palavra-chave "carbon nanotubes" apresentou 110.726 documentos. Levando-se em conta que o mais antigo é datado de 1992, temos o correspondente, em média, a 5033 artigos científicos/ano (o que não corresponde à realidade, pois o número de artigos cresce a cada ano - foram 14.704 em 2012). Somente para comparação, o número total de resultados para a mesma busca utilizando a palavra chave "graphite" ou "diamond" apresenta respectivamente 162.697 e 120.888 resultados, sendo que os mais antigos são dos anos de 1864 e 1865 , respectivamente.

O mais recente alótropo de carbono, também representante da família dos nanomateriais, é exatamente o grafeno (Figura 1f). Conhecido como a unidade básica estrutural cujo empilhamento origina a estrutura do grafite, o grafeno isolado corresponde a um material bidimensional com a espessura monoatômica, que vinha sendo estudado por grupos de cientistas teóricos há mais de 50 anos. Tido como impossível de ser obtido em sua forma livre, o grafeno foi isolado e caracterizado pela primeira vez em 2004, através de sucessivas etapas de peeling de um pedaço de grafite,${ }^{15}$ com o auxílio de uma fita adesiva, trabalho que rendeu o prêmio Nobel de física de 2010 a seus autores, A. Geim e K. Novoselov, da Universidade de Manchester. O grafeno foi o primeiro cristal bidimensional estável isolado, e seu longo sistema $\pi$ conjugado, onde os elétrons estão confinados em duas dimensões, lhe confere propriedades excepcionais: ${ }^{16}$ os elétrons no grafeno se comportam como partículas relativísticas de massa zero, o que acarreta em um efeito de Quantum Hall bastante anômalo, alta mobilidade eletrônica, e condutividade térmica e resistência mecânica similares às dos nanotubos. Assim como os nanotubos de carbono, o termo grafeno hoje corresponde a uma família de compostos, desde a folha monoatômica, até materiais formados por duas, três, quatro, etc (até dez) folhas de grafeno empilhadas de forma organizada. Estes materiais, conhecidos como grafeno mono-camada, grafeno bi-camada, etc, têm propriedades eletrônicas distintas, devido aos diferentes acoplamentos eletrônicos entre as várias folhas em cada uma das estruturas. Embora seja o mais novo membro da família dos 
alótropos do carbono, com menos de 10 anos de existência, o grafeno já vem sendo considerado, assim como os nanotubos de carbono, um material altamente estratégico, com inúmeras possibilidades reais de aplicações.

\section{PREPARAÇÃO, PROCESSAMENTO, DESAFIOS ATUAIS E QUÍMICA}

Embora existam alguns métodos de preparação de nanotubos de carbono, o método de deposição química de vapor (CVD - chemical vapor deposition) possibilita nos dias atuais a preparação de nanotubos de carbono em grande escala. $\mathrm{O}$ método é baseado na decomposição de precursores de carbono (hidrocarbonetos, álcoois, etc.) em fornos com temperatura e atmosfera controlados, sobre catalisadores metálicos adequados. ${ }^{9} \mathrm{O}$ avanço na compreensão das etapas de crescimento de nanotubos de carbono via processo CVD, resultante da evolução das técnicas de caracterização temporais e in situ (principalmente técnicas de microscopia eletrônica), possibilitou um controle bastante elevado na homogeneidade das amostras preparadas, com um controle na produção de amostras de nanotubos de paredes múltiplas ou paredes simples, e neste último caso, amostras semicondutoras ou metálicas. ${ }^{17}$ Além disso, hoje já é possível a preparação de amostras com um alto grau de alinhamento entre os tubos (uns em relação aos outros). A preparação de amostras de nanotubos de parede simples com um rigoroso controle no diâmetro e na quiralidade ainda é um desafio, embora já existam relatos nesta direção. ${ }^{18}$

Para várias aplicações, os nanotubos necessitam estar dispersos, de forma estável, em diferentes tipos de solventes. Grandes avanços foram realizados, através de combinações entre tratamentos químicos e utilização de moléculas surfactantes. Estas combinações vêm sendo utilizadas, também, para separar amostras de nanotubos com diferentes quiralidades, a partir de interações seletivas com diferentes substâncias. ${ }^{19}$

O papel da química no processo de crescimento de nanotubos de carbono é absolutamente central - não obstante o método de preparação contém "química" em seu nome. Entretanto, a importância da química vai muito além da compreensão do processo de conversão do precursor ao nanotubo, atuando também como atriz principal nos processos de purificação, processamento e separação de nanotubos. Dentre algumas ações, a química é diretamente responsável por: i) a preparação de nanopartículas estáveis de catalisador, com controle de tamanho, homogeneidade, composição, cristalinidade e alinhamento, cruciais para a qualidade das amostras obtidas; ii) as etapas de tratamento pós-síntese para remoção de catalisadores e possíveis sub-produtos da síntese; iii) a preparação de dispersões estáveis de nanotubos, por meio de tratamentos químicos e/ou interações com diferentes surfactantes; iv) os processos de separações seletivas de nanotubos de diferentes quiralidades, por técnicas como centrifugação por densidade de gradiente ou cromatografia a gel; v) as modificações estruturais e funcionalizações de nanotubos, tanto nas paredes externas como no interior das suas cavidades; vi) o desenvolvimento e aprimoramento de várias técnicas de caracterização.

No que diz respeito ao grafeno, o principal desafio ainda está no desenvolvimento de métodos massivos de produção de amostras com qualidade estrutural, e com controle do número de camadas. Dentre os métodos de produção atuais, o peeling do grafite com fita adesiva proporciona amostras com qualidade estrutural, de forma simples, mas com baixíssimo rendimento - o que o inviabiliza para quaisquer possibilidades de aplicação. O método CVD, sob condições parecidas às utilizadas para o crescimento de nanotubos, mas utilizando-se substratos metálicos, possibilita o crescimento de monocamadas com baixo número de defeitos, mas também não corresponde, neste caso, a uma técnica de produção massiva (embora adequada para aplicações em microeletrônica, por exemplo). ${ }^{20}$ Dentre as alternativas apresentadas até o momento, grandes quantidades de amostra podem ser preparadas pela exfoliação do grafite, ${ }^{21,22}$ e neste caso o método mais abundante é totalmente dependente de rotas químicas, ${ }^{23}$ como ilustrado esquematicamente na Figura 2. Esta rota consiste em três etapas distintas, iniciando pela oxidação química do grafite a óxido de grafite, na qual vários átomos de carbono $\mathrm{sp}^{2}$ são oxidados a $\mathrm{sp}^{3}$, com a adição de grupamentos funcionais oxigenados; a seguir realiza-se a exfoliação do óxido de grafite em óxido de grafeno, pela separação das folhas bidimensionais contendo os grupamentos oxigenados; e finalmente o óxido de grafeno é quimicamente reduzido a grafeno, por meio de agentes redutores como hidrazina. ${ }^{23}$ Apesar de produzir quantidades razoáveis de material, vários grupamentos oxigenados permanecem na estrutura do grafeno, além de defeitos estruturais na rede, o que significa amostras com baixa qualidade estrutural.

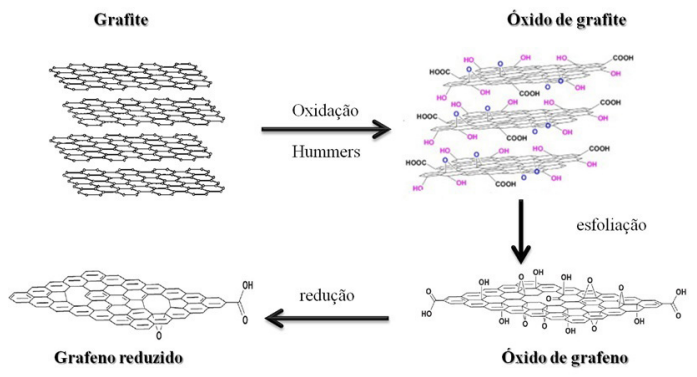

Figura 2. Representação esquemática do processo de oxidação do grafite para formação do óxido de grafite, seguido da exfoliação para formação de folhas individuais de óxido de grafeno, seguido de redução para formação de grafeno (ou óxido de grafeno reduzido)

Uma nova vertente que vem crescendo nos anos recentes consiste na síntese total de grafeno, a partir de precursores moleculares como o benzeno, o que representa em um desafio extremamente importante para químicos sintéticos. . $^{24,25}$

A maior necessidade atual da área, entretanto, diz respeito à toxicidade dos nanotubos de carbono e do grafeno. Uma vez que já estão no mercado, e em vários produtos comerciais (como será discutido adiante), informações relacionadas à toxicidade destes materiais são fundamentais para que sua utilização e descarte seguros, tanto para os seres humanos quanto para o meio ambiente, sejam garantidos.

A nanotoxicologia é um ramo que utiliza conceitos da nanobiociência para a compreensão dos impactos tóxicos potenciais dos nanomateriais nos sistemas biológicos. O primeiro relato da palavra nanotoxicologia no portal Web of Science data de 2004, e trata-se de um artigo da revista Science cujo título "Nanotoxicology: Nanotechnology grows up" deixa claro o que viria a ser uma das grandes preocupações vinculadas à utilização, manipulação e descarte dos nanomateriais, ${ }^{26}$ e a necessidade do desenvolvimento de uma avaliação das implicações da exposição aos nanomateriais, como dados relacionados à sua adsorção, distribuição, metabolismo e excreção nos sistemas biológicos. Além disso, informações relacionadas ao efeito do tamanho das partículas e distribuição de tamanho são fundamentais para o entendimento de como os nanomateriais são distribuídos e eliminados nos organismos vivos. ${ }^{27}$

Assim como ocorre com suas propriedades, a toxicidade potencial dos nanotubos de carbono é fortemente correlacionada com sua estrutura, quiralidade, comprimento, área superficial, possível contaminação metálica com catalisadores e a funcionalização ou não da sua superfície. ${ }^{28}$ Hoje sabe-se que a adição de nanotubos de carbono pode iniciar ou mesmo acelerar várias atividades intracelulares em organismos biológicos, tais como processos inflamatórios, genotoxicidade e/ou atividade carcinogênica. ${ }^{28}$ 
Com relação à toxicidade intrínseca, espera-se que materiais ideais baseados em carbono não sejam tóxicos, em função de sua baixa reatividade química e bioquímica em condições normais de pressão e temperatura. ${ }^{28}$ Entretanto, com a sua funcionalização, com a presença de defeitos estruturais e impurezas (resíduos de catalisador, por exemplo), existe a possibilidade da ocorrência de novos caminhos para reações bioquímicas. Sabe-se ainda que a alta razão de aspecto e o alto módulo de Young desencadeiam uma série de mecanismos que podem levar à deterioração das células e ativar uma resposta tóxica intensa. ${ }^{28,29}$ Estudos demostram certo grau de atividade inflamatória devido à presença de nanotubos de carbono, mas as características carcinogênicas dos mesmos ainda está em debate, sendo alvo de forte controvérsia. ${ }^{30}$ Sabe-se também que nanotubos de carbono e grafeno com suas superfícies funcionalizadas são excretados mais facilmente dos organismos quando comparados aos materiais da forma que são obtidos, uma vez que, por serem compatíveis com fluídos biológicos, sua excreção via rota renal é permitida, o que minimiza sua bioacumulação. ${ }^{31}$

Além da toxicidade intrínseca, outros parâmetros devem ser considerados, tais como biodegradabilidade e biopersistência, influência das alterações morfológicas (incluindo transições conformacionais), bem como os efeitos do tamanho. ${ }^{27}$ Atualmente as preocupações mais importantes estão relacionadas com a estabilidade e os efeitos potenciais do ciclo de vida dos nanomateriais de carbono sobre animais, seres humanos e meio ambiente. ${ }^{32}$ Entretanto, dados experimentais ainda são bastante controversos, com diversas questões ainda em aberto.

Apesar das dificuldades relacionadas à avaliação da toxicidade de materiais baseados em carbono, sabe-se que eles têm um enorme potencial para serem aplicados no tratamento e no diagnóstico de doenças, no campo chamado de Nanomedicina. Assim, estudos relacionados à sua biodegradação apresentam dados promissores no que diz respeito ao seu uso e sua biopersistência e acumulação. ${ }^{33,34}$

\section{DA APLICAÇÃO EM POTENCIAL AOS PRODUTOS REAIS}

Uma busca na vasta literatura envolvendo nanotubos de carbono e grafeno possibilita um levantamento abundante sobre aplicações em potencial destes materiais em várias áreas, e algumas delas serão brevemente sumarizadas a seguir, com destaque maior para os nanotubos de carbono:

i) compósitos: sem dúvida a maior possibilidade de aplicação dos nanotubos de carbono (do tipo multi-paredes) está na área de materiais compósitos, principalmente com polímeros. ${ }^{35} \mathrm{~A}$ alta razão de aspecto (relação comprimento/diâmetro) dos nanotubos faz com que o limite de percolação destes dispersos em matrizes poliméricas seja extremamente baixo. A introdução de quantidades variáveis (de $0,01 \%$ em massa até $20 \%$ ) de nanotubos em polímeros leva a materiais com aumento da resistência mecânica e química, aumento de condutividade e melhora nas propriedades térmicas. ${ }^{36}$ Em compósitos também está uma das maiores promessas de aplicações de grafenos, ${ }^{16,25}$

ii) energia: nanotubos de carbono vêm sendo utilizados em diversos aspectos relacionados ao armazenamento, conversão e transmissão de energia elétrica. ${ }^{17}$ Nanotubos multi-paredes são extensivamente usados em baterias de íons lítio para notebooks e telefones celulares, misturados aos materiais do cátodo e do ânodo, aumentando a condutividade elétrica e a resistência mecânica, o que aumenta o ciclo de vida das baterias, ${ }^{37}$ nanotubos de carbono (parede única ou múltipla) vêm sendo usados, puros ou em diferentes compósitos, em supercapacitores, incluindo supercapacitores flexíveis, com altíssima performance; ${ }^{38}$ nanotubos de carbono atuam como materiais para a camada transportadora de elétrons em células solares orgânicas e flexíveis, aumentando o rendimento destes dispositivos; $;^{39}$ nanotubos de carbono vêm sendo continuamente empregados como suporte para catalisadores (principalmente platina) em células a combustível, permitindo um aumento na condutividade e uma redução da quantidade de platina no dispositivo, diminuindo seu custo. ${ }^{40}$ Em todas estas aplicações, já existem vários relatos, com performance bastante adequadas, utilizando-se também o grafeno; ${ }^{16,25}$

iii) sensores e biosensores: por ter suas propriedades fortemente dependentes do ambiente, nanotubos de carbono (tanto de paredes simples como de paredes múltiplas) e grafeno vêm sendo utilizados como sensores extremamente sensíveis aos mais diversos analitos, incluindo biomoléculas. ${ }^{41,42}$ Variações na condutividade, fluorescência, propriedades óticas, impedância, piezoeletricidade, mudanças espectroscópicas, dentre outras, em função da adsorção de moléculas alvo nas paredes dos nanotubos ou na superfície do grafeno, são facilmente detectáveis, o que acarreta em sensores com baixíssimos limites de detecção e alta seletividade. Sensores para gases, toxinas, fragmentos de DNA, os mais diversos tipos de biomoléculas, fármacos, dentre muitos outros, vêm sendo continuamente descritos na literatura;

iv) eletrônica: nanotubos de carbono de paredes simples, com um elevado controle de quiralidade e alta pureza, são candidatos ideais para transistores (FET - field-efect transistors), com performances superiores aos encontrados para dispositivos baseados em silício. ${ }^{43}$ Estes dispositivos são construídos baseados tanto em nanotubos individuais (embora as dificuldades na preparação de nanotubos de paredes simples com o rigoroso controle de quiralidade, aliado ao alto preço deste tipo de nanotubo, seja um limitante importante para viabilização de produção em larga escala), como em arranjos de nanotubos; nanotubos de paredes múltiplas também são candidatos a substituir o cobre em conexões em placas e dispositivos em microeletrônica, diminuindo as perdas e aumentando a capacidade de corrente $;{ }^{17}$ nanotubos também estão associados como materiais para dissipar calor em circuitos eletrônicos. Com relação ao grafeno, talvez a aplicação em eletrônica, principalmente em FET, seja a mais próxima de uma aplicação real, utilizando-se grafeno crescido por CVD como componente ativo, ${ }^{44}$

v) filmes finos: uma das aplicações mais divulgadas nos últimos anos para nanotubos de carbono, grafeno e seus diferentes nanocompósitos (incluindo com polímeros condutores e nanofios metálicos) está na preparação de eletrodos transparentes condutores, visando substituir o ITO (óxido de índio dopado com óxido de estanho), para utilização em touch screens, LED's e OLED's, células solares flexíveis, entre outros; ${ }^{45-47}$ filmes para recobrimento e proteção anti-corrosão também encontram forte apelo de aplicação para estes materiais, ${ }^{17}$

vi) meio ambiente: diversas aplicações de nanotubos de carbono em processos de purificação e descontaminação de águas (filtros e membranas), ${ }^{48}$ ou como foto- e eletro-catalisador para oxidação de contaminantes vêm sendo reportados, ${ }^{49}$

vii) catálise: nanotubos de carbono e grafeno são materiais ideais para suporte de catalisadores (óxidos, metálicos ou mesmo moleculares); várias reações são também catalisadas pelo próprio nanotubo ou grafeno..$^{50}$

Os pontos brevemente sumarizados nos parágrafos anteriores correspondem a uma fração das aplicações em potencial destes materiais. Várias outras, incluindo muitas aplicações não-convencionais, têm sido diariamente descritas na literatura. Entretanto, quantas destas aplicações se transformaram - ou virão a se transformar - em produtos reais? A resposta desta pergunta começa pelo conhecimento de alguns números: estima-se que a produção anual de nanotubos de carbono, 
em 2013, está na faixa de 4000 toneladas/ano - um aumento de mais de 10 vezes em relação à produção do ano de $2006 .{ }^{17}$ De Volder e colaboradores, em um trabalho recente publicado no periódico Science ${ }^{17}$ listam 33 empresas que produzem e comercializam grandes quantidades de nanotubos de carbono (incluindo gigantes como a Mitsubishi), concentradas nos Estados Unidos, Alemanha, França, China, Austrália, Índia, Coréia do Sul, Japão, Canadá, Bélgica, Grécia, e Inglaterra. O mercado global de nanotubos de carbono em 2011 foi de US\$ 192 milhões, com estimativa ${ }^{51}$ para US\$ 527 milhões em 2016 (mesmo considerando o recente anúncio de que a produção de nanotubos de carbono pela Bayer foi interrompida em 2013. Os motivos desta interrupção, entretanto, não estão relacionados a perspectivas de perda de mercado). Os custos diminuíram sensivelmente nos últimos anos, e dependem do tipo e da qualidade dos nanotubos, variando de US\$ 30 a US\$ 75 o quilo de nanotubos multi-paredes, aumentando para nanotubos de paredes simples, e se tornando mais caro quanto mais específica, controlada e pura for a amostra.

Os números descritos acima não deixam dúvida de que se trata de um material que veio para ficar. Hoje em dia já existem mais de 50 empresas mundo afora comercializando mais de 150 produtos contendo nanotubos de carbono. Dentre eles, destacam-se vários compósitos utilizados em produtos esportivos, como solado de tênis de corrida (comercializado pela Adidas), tacos de golfe (Aldila), tacos de beisebol (Easton), bicicletas (BMC), raquetes de tênis e de badminton (Yonex) e flechas para arco e flecha (Easton); compósitos estruturais em cascos de iates (Baltic) e lanchas (Zyvex); compósitos para turbinas de captação de energia eólica (Amroy, Hexcel e Eagle Windpower); compósitos para linha de combustível em automóveis, e para tinta eletrostática (Hyperion catalysis); compósitos para fios, fitas e adesivos (Meijo-nano carbon); compósitos para proteção contra interferência eletromagnética em automóveis, aviões, satélites e veículos espaciais, para proteção contra faísca e retardante de chama (Nanocomp); compósitos em coletes a prova de bala (Nanocomp); compósitos para proteção contra interferência eletromagnética em embalagens eletrônicas (Nanocyl, Axson); compósitos para componentes de máquina e para tecnologia aeroespacial (Zoz GmbH); plásticos condutores para linhas de combustível e indústria automotiva (BASF e Audi); sensores para diagnóstico de doenças (Aneeve, NanoIntegris, Nanomix, Nantero,); circuitos impressos e transistores (Intel, NEC Corp., Paru Corp.), baterias (ANS, BlueNano, Showa Denko, Ube); eletrodos transparentes condutores, touch screens, touch sensors (Canatu, Eikos, Iijin Nanotech, NanoIntegris, Nokia, Toray, Panasonic, Samsung, Teco Nanotech Co.,Top Nanosys, Unidym); televisores com tela emissora de elétrons de condução de superfície (Canon); sensores térmicos (GE); membranas de filtração (NanOasis, Porifera); memórias não-voláteis (Nantero); sistemas de purificação de água (Seldon); janelas com dissipação eletrostática (Takiron Co.); dispositivos fotovoltaicos orgânicos (Unidym Inc.), dentre outros.

Somente uma empresa chinesa, a CNTouch, ${ }^{52}$ produz $1,5 \mathrm{mi}-$ lhão de painéis sensíveis ao toque (touch panels) a cada mês (dado atualizado em junho/2013), totalmente baseados em nanotubos de carbono. Estes painéis podem ser encontrados nos telefones celulares e tablets comercializados atualmente pela Philips, Huawei, Coolpad, TCL, Nabi e MiO.

\section{E NO BRASIL?}

Acompanhando a tendência mundial, a área de N\&N experimentou um crescimento significativo no Brasil, o que pode ser comprovado pelo número de artigos, número de pesquisadores e número de grupos de pesquisa relacionados à área. Dos 948.444 artigos no Web of Science oriundo da busca com a palavra-chave nano* (junho/2013), 10.096 têm autoria de brasileiros, o que corresponde a 1,06\% da produção total. A química, neste contexto, aparece como a área do conhecimento no Brasil com maior número de pesquisadores e grupos de pesquisa. A plataforma Lattes do CNPq lista 1663 pesquisadores doutores associados a alguma atividade em nano, sendo destes 709 na química $(42,6 \%)$ e 460 na física $(27,7 \%)$. No que diz respeito ao diretório dos grupos de pesquisa do $\mathrm{CNPq}$, estes números são também bastante significativos, e rumam na mesma direção: um total de 586 grupos de pesquisa com atividades em nano (palavra-chave para busca = nanomateriais ou nanopartículas ou nanotecnologia ou nanotubos), sendo 171 na química, 163 nas engenharias e 96 na física. Uma forma de se avaliar o impacto desta área na comunidade científica da química brasileira pode ser por meio dos trabalhos submetidos à Reunião Anual da Sociedade Brasileira de Química (RASBQ). Analisando a evolução do percentual de trabalhos em N\&N apresentados nas últimas cinco RASBQs (em relação ao total de trabalhos), observa um crescimento constante, saindo de 4,4\% em 2009, 6,5\% em 2010 e 2011 e 7,9\% em 2012. Em 2013, uma fração de 9,1\% de todos os trabalhos submetidos à RASBQ foi em N\&N, distribuídos em 12 (das 15) divisões da química. Para um evento que envolve todas as áreas da química, estes números são bastante significativos.

Este panorama que se observa para a N\&N em geral também se aplica nas nanoestruturas de carbono. Indiscutivelmente, o Brasil faz parte do mapa global da pesquisa em nanotubos de carbono e grafeno, com vários grupos reconhecidos e consolidados. Um dos Institutos Nacionais de Ciência e Tecnologia (INCT) financiados pelo Ministério da Ciência e Tecnologia do Brasil se dedica exclusivamente aos diferentes aspectos relacionados à síntese, caracterização, aplicações, e estudo de toxicologia e impactos na saúde de nanotubos, grafeno e fulerenos (INCT de nanoestruturas de carbono). ${ }^{53}$ Este INCT agrega 54 pesquisadores, de diferentes formações e especialidades (químicos, físicos, biólogos, engenheiros) de 18 instituições de pesquisa e 2 empresas de oito diferentes estados no Brasil. Além deste, vários outros INCTs têm como foco algum tipo de pesquisa e aplicação envolvendo nanoestruturas de carbono.

O Web of Science lista 1209 trabalhos com as palavras chave nanotubos de carbono tendo o Brasil como endereço dos autores - isso corresponde a 1,09\% da produção total de artigos em nanotubos de carbono nesta base de dados. Pesquisa similar aponta 400 trabalhos em grafeno, o que corresponde a 1,23\% da produção total. A química também é predominante entre os pesquisadores e grupos de pesquisa brasileiros em nanotubos de carbono: a plataforma Lattes lista 756 pesquisadores doutores, sendo 431 na química $(57,0 \%)$ e 223 na física $(29,5 \%)$, e o diretório de grupos de pesquisa lista 91 grupos no total, sendo 30 na química e 27 na física. A distribuição regional, as instituições e o número de grupos por instituições, em nanotubos de carbono no Brasil, estão ilustrados na Figura 3. Em grafeno, a física se sobrepõe: dos 223 pesquisadores listados na plataforma Lattes, 105 são da física $(47,1 \%)$ e 67 da química $(30,0 \%)$.

Embora tenha destaque no quesito ciência, o país está muito atrasado em relação a patentes e a tecnologia. A base de dados do INPI registra apenas 57 patentes contendo nanotubos de carbono no título ou resumo, e míseras 3 ocorrências com grafeno. Dados não-oficiais estimam que o Brasil hoje produz aproximadamente 300 produtos de base nanotecnológica (este número é bastante contraditório, podendo chegar a 700), e aparentemente nenhum baseado em nanoestruturas de carbono. Além disso, se alguma grande indústria nacional tiver interesse em incorporar nanotubos de carbono em seus produtos, ou mesmo desenvolver novos produtos baseados em nanotubos, necessariamente terá que importar este insumo. Enquanto países desenvolvidos (como EUA, Japão e Inglaterra) e em desenvolvimento (como a China e Índia) produzem mensalmente toneladas de nanotubos de carbono, a produção de nanotubos de carbono no Brasil está restrita a poucos laboratórios de universidades e institutos de pesquisa. Esta 


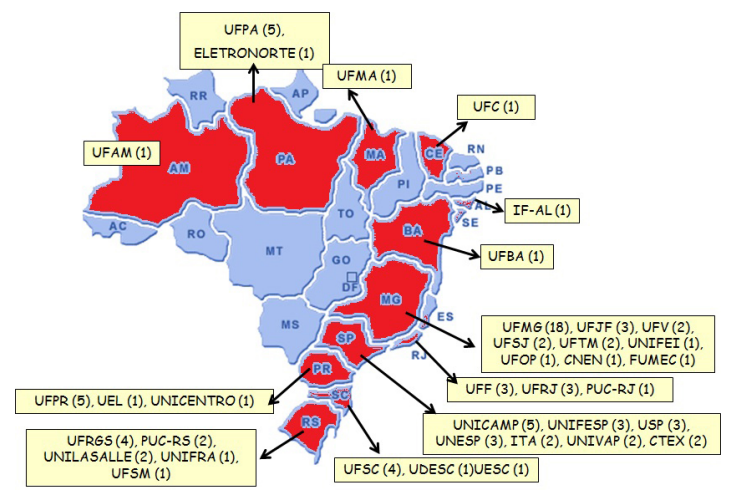

Figura 3. Distribuição regional dos grupos de pesquisa em nanotubos de carbono no país. Os números entre parêntesis correspondem ao número de grupos cadastrados em cada instituição (fonte: diretório dos grupos de pesquisa do $C N P q$ )

realidade deve mudar em breve, com a efetivação do CT-Nanotubos, um projeto de pesquisadores da UFMG, com apoio da Petrobras, BNDES, e dos governos municipal de Belo Horizonte e estadual de Minas Gerais, que visa a construção, no parque tecnológico de Belo Horizonte (BH-Tec), de um espaço destinado à fabricação em escala pré-industrial de nanotubos de carbono.

\section{CONSIDERAÇÕES FINAIS}

Passados 22 anos de sua primeira descrição, os nanotubos de carbono deixaram de ser uma promessa em potencial e atingiram o último estágio do desenvolvimento tecnológico, sendo comercializados como componentes de diversos produtos, dispositivos e sistemas. Apesar disso, ainda há muito que ser compreendido, e o interesse científico e tecnológico relacionado a este material fascinante está bem distante da estagnação, o que pode facilmente ser conferido pelo número crescente de trabalhos científicos publicados diariamente, onde novas aplicações, novas propriedades e novas soluções para problemas antigos são continuamente descobertas. Muitos dos mitos e lendas dos primórdios dos nanotubos de carbono foram gradativamente sendo derrubados, como a suposta dificuldade de produção em grande escala, a suposta impossibilidade na obtenção de amostras puras e controladas, e os supostos altos preços, que inviabilizariam sua aplicação. Esta realidade só foi possível devido à alta inter- e multidisciplinaridade da área, onde as fronteiras clássicas de disciplinas foram rompidas e novas concepções do fazer ciência foram adotadas. Indiscutivelmente, a química teve um papel central neste processo passado, e certamente continuará tendo nas etapas futuras.

Com relação ao grafeno, trata-se de um pré-adolescente com 9 anos de idade, que se beneficiou muito da existência do irmão mais velho. Muito do conhecimento desenvolvido para os nanotubos foi adaptado para o grafeno e o caminho das pedras certamente se tornou menos inóspito. O estágio de desenvolvimento atual dos diferentes aspectos relacionados ao grafeno é bem maior que aqueles relacionados aos nanotubos de carbono no ano 2000 (quando tinha a mesma idade do grafeno hoje), o que pode nos fazer inferir que o estágio de maturidade do grafeno se dará em um tempo menor. Hoje, o maior desafio da área consiste em preparar amostras estruturalmente perfeitas, com grandes tamanhos de folhas, com controle no número de folhas empilhadas (mono-, bi-, tr-camadas, etc.), e fundamentalmente, em grande quantidade. Novamente, a química adota um papel de destaque nos avanços alcançados até o momento e naqueles que naturalmente virão.

A velocidade com que os avanços descritos anteriormente se deram não foi, infelizmente, acompanhada pelas questões de saúde pública e de controle ambientais relacionados à manipulação, armazenamento, contato, descarte, etc. dos nanomateriais de carbono. A grande heterogeneidade e complexidade destes sistemas, aliada a certa demora em se iniciar estudos sistemáticos em toxicologia destes nanomateriais, vêm produzindo dados científicos ainda conflitantes e contraditórios. Esta realidade se torna mais grave quando sabemos que já há no mercado vários produtos baseados em nanotubos de carbono. Felizmente, grandes ações globais em diferentes países começam a tentar suprir esta carência.

Há alguns anos, nos primórdios dos nanotubos de carbono, ficou famosa uma correspondência recebida por uma pesquisadora brasileira, em resposta a uma solicitação para comprar nanotubos de carbono de uma empresa americana. Nesta correspondência, a empresa se desculpava por não poder vender este produto a consumidores no Brasil, por se tratar de um material estratégico que era regulado pelo governo norte-americano. Naquele momento, tratava-se apenas de um material em potencial, e os cientistas brasileiros se deram conta da enorme necessidade em se produzir nanotubos de carbono no país. Nos dias atuais, aquela realidade parece história de ficção científica: vários países, incluindo dois do bloco do BRIC (China e Índia), produzem nanotubos de carbono em quantidade, qualidade e baixo custo, que podem ser adquiridos como matéria prima para a indústria. No Brasil, a produção acadêmica na área cresce continuamente (incluindo contribuições importantes na área de toxicologia), em um ritmo que, infelizmente, não é acompanhado pelo setor industrial. A perspectiva da efetivação de um polo para o início de uma produção em escala de nanotubos, com apoio de uma grande petrolífera, pode ser um alento nesta direção. Iniciativas nesta direção precisam ser incentivadas, multiplicadas e rapidamente efetivadas, sob o risco de ficarmos (enquanto nação) novamente dependentes em uma área extremamente soberana e estratégica.

\section{AGRADECIMENTOS}

Os autores agradecem os Editores deste número especial pelo convite; ao CNPq, CAPES, NENNAM (PRONEX-Fundação Araucária/CNPq) e ao INCT de Nanomateriais de Carbono pelo auxílio financeiro.

\section{REFERÊNCIAS}

1. Zarbin, A. J. G.; Quim. Nova 2007, 30, 1469.

2. Dresselhaus, M. S.; Dresselhaus, G.; Eklund, P. C.; Science of Fullerenes and Carbon Nanotubes. Academic Press: San Diego, 1995.

3. Kroto, H. W.; Heath, J. R.; O’Brien, S. C.; Curl, R. F.; Smalley, R. E.; Nature 1985, 318, 162.

4. Santos, L. J.; Rocha, G. P.; Alves, R. B.; Freitas, R. P.; Quim. Nova 2010, 33,680 .

5. Radushkevich, L. V.; Lukyanovich, V. M.; Zurn. Fisic. Chim. 1952, 26, 88 .

6. Oberlin, A.; Endo, M.; Koyama, T.; J. Cryst. Growth 1976, 32, 335.

7. Iijima, S.; Nature 1991, 354, 56.

8. Ajayan, P. M.; Iijima, S., Nature 1992, 358, 23.

9. Terrones, M.; Annu. Rev. Mater. Res. 2003, 33, 419.

10. Charlier, J-C.; Blase, X.; Roche, S.; Rev. Mod. Phys. 2007, 79, 677.

11. Wepasnick, K. A.; Smith, B. A.; Schrote, K. E.; Wilson, H. K.; Diegelmann, S. R.; Fairbrother, D. H.; Carbon 2011, 49, 24.

12. Moraes, R. A.; Matos, C. F.; Castro, E. G.; Schreiner, W. H.; Oliveira, M. M.; Zarbin, A. J. G.; J. Braz. Chem. Soc. 2011, 22, 2191.

13. Schnitzler, M. C.; Oliveira, M. M.; Ugarte, D.; Zarbin, A. J. G.; Chem. Phys. Lett. 2003, 381, 541.

14. Araujo, A. E. P.; Duque, J. G. S.; Knobel, M.; Schnitzler, M. C.; Zarbin, A. J. G.; J. Magn. Magn. Mater. 2007, 312, 32. 
15. Novoselov, K. S.; Geim, A. K.; Morozov, S. V.; Jiang, D.; Zhang, Y.; Dubonos, S. V.; Grigorieva, I. V.; Firsor, A. A.; Science 2004, 306, 666.

16. Singh, V.; Joung, D.; Zhai, L.; Das, S.; Khondaker, S. I.; Seal, S.; Prog. Mater. Sci. 2011, 56, 1178.

17. De Volder, M. F. L.; Tawfick, S. H.; Baughman, R. H.; Hart, A. J.; Science 2013, 339, 535

18. Liu, J.; Wang, C.; Tu, X. M.; Liu, B. L.; Dren, L.; Zheng, M.; Zhou, C. W.; Nat. Commun. 2012, 3, 1199.

19. Arnold, M. S.; Green, A. A.; Hulvat, J. F.; Stripp, S. I.; Hersam, M. C.; Nat. Nanotechnol. 2006, 1, 60.

20. Li, X.; Cai, W.; An, J.; Kim, S.; Nah, J.; Yong, D.; Piner, R.; Veldmakanni, A.; Juerg, I.; Tutuk, E.; Banerjee, S. K.; Colombo, L.; Ruoff, R. S.; Science 2009, 324, 1312.

21. Vallés, C.; Drummond, C.; Saadaoui, H.; Furtado, C. A.; He, M.; Roubean, D.; Ortolani, L.; Monthioux, M., Penicaud, A.; J. Am. Chem. Soc. 2008, 130, 15802.

22. Salvatierra, R. V.; Domingues, S. H.; Oliveira, M. M.; Zarbin, A. J. Z.; Carbon 2013, 57, 410.

23. Park, S.; Ruoff, R. S.; Nat. Nanotechnol. 2009, 4, 25.

24. Wu, W. S.; Pisula, W.; Müllen, K.; Chem. Rev. 2007, 107, 718.

25. Luo, B.; Liu, S.; Zhi, L.; Small 2012, 8, 630.

26. Service, R. F.; Science 2004, 304, 1732.

27. Thomas, K.; Sayre, P.; Toxicol. Sci. 2005, 87, 316.

28. Popescu, B. M.; Ali, N.; Basturea, G.; Comsa, G. I.; Materon, L. A.; Chipara, M.; Appl. Surf. Sci. 2013, 275, 2.

29. Donaldson, K.; Aitken, R.; Tran, L.; Stone, V.; Duffin, R.; Forrest, G.; Alexander, A.; Toxicol. Sci. 2006, 92, 5.

30. Katwa, P.; Wang, X. J.; Urankar, R. N.; Podila, R.; Hilderbrand, S. C.; Fick, R. B.; Rao, A. M.; Ke, P. C.; Wingard, C. J.; Brown, J. M.; Small 2012, 8, 2904.

31. Lacerda, L.; Bianco, A., Prato, M.; Kostatarelos, K., Adv. Drug Delivery Rev. 2006, 58, 1460.

32. Kahru, A.; Ivask, A.; Acc. Chem. Res. 2013, 46, 823.

33. Johnston, H.; Brown, D.; Kermanizadeh, A.; Gubbins, E.; Stone, V.; J. Controlled Release 2012, 164, 307.
34. Seabra, A. B.; Paula, A. J.; Durán, N.; Biotechnol. Progr. 2013, $29,1$.

35. Byrne, M. T.; Gun'ko, Y. K.; Adv. Mater. 2010, 22, 1672.

36. Matos, C. F.; Galembeck, F.; Zarbin, A. J. G.; Carbon 2012, 50, 4685.

37. Dai, L.; Chang, D. W.; Baek, J-B; Lu, W.; Small 2012, 8, 1130.

38. King, P. J.; Higgins, T. M.; De, S.; Nicoloso, N.; Coleman, J. N.; ACS Nano 2012, 6, 1732.

39. Canestraro, C. D.; Schnitzler, M. C.; Zarbin, A. J. Z.; Luz, M. G. E.; Roman, L. S.; Appl. Surf. Sci. 2006, 252, 5575.

40. Yang, Z.; Nie, H. G.; Chen, X.; Chen, X. H.; Huang, S. M.; J. Power Sources 2013, 236, 238.

41. Cava, C. E.; Salvatierra, R. V.; Alves, D. C. B.; Ferlauto, A. S.; Zarbin, A. J. G.; Roman, L. S.; Carbon 2012, 50, 1953.

42. Souza, L. P.; Calegari, F.; Zarbin, A. J. G.; Marcolino-Junior, L. H.; Bergamini, M. F.; J. Agric. Food Chem. 2011, 59, 7620.

43. Franklin, A. D.; Luisier, M.; Han, S. J.; Tulevski, G.; Breslin, C. M.; Gignac, L.; Lundstom, M. S.; Haensdi, W.; Nano Lett. 2012, 12, 758.

44. Wei, D. C.; Wu, B.; Guo, Y. L.; Yu, G.; Liu, Y. Q.; Acc. Chem. Res. 2013, $46,106$.

45. Salvatierra, R. V.; Cava, C. E.; Roman, L. S.; Zarbin, A. J. G.; Adv. Funct. Mater. 2013, 23, 1490.

46. Domingues, S. H.; Salvatierra, R. V.; Oliveira, M. M.; Zarbin, A. J. G.; Chem. Commun. 2011, 47, 2592.

47. Kholmanov, I. N.; Domingues, S. H.; Chou, H.; Wang, X.; Tan, C.; Kim, J-Y.; Li, H.; Piner, R.; Zarbin, A. J. G.; Ruoff, R. S.; ACS Nano 2013, 7, 1811.

48. Gupta, V. K.; Saleh, T. A.; Environ. Sci. Pollut. Res. 2013, 20, 2828.

49. An, X. Q.; Yu, J. C.; RSC Adv. 2011, 1, 1426.

50. Zhu, J.; Holmen, A.; Chen, D.; ChemCatChem 2013, 5, 378.

51. http://www.bccresearch.com/market-research/nanotechnology/carbonnantubes-markets-technologies-nan024e.html, acessada em Julho 2013.

52. http://www.cntouch.com, acessada em Julho 2013.

53. http://www.nanocarbono.net, acessada em Julho 2013. 